\title{
Antibacterial Activity of Chitosan on Some Common Food Contaminating Microbes
}

\author{
Nguyen Van Toan*, Tran Thi Hanh and Pham Vo Minh Thien
}

\author{
School of Biotechnology, International University, Ho Chi Minh, Vietnam, Vietnam National University, Ho Chi Minh \\ City, Vietnam
}

\begin{abstract}
The antimicrobial activity of chitosan was thoroughly investigated. The prepared chitosan solutions at diluted concentrations and in aerosol form, of which applied to apples and bananas had considerably prolonged the storage time of the fruits and prevented them from microbes contaminating and growing. The study has reconfirmed that chitosan exhibits high antimicrobial activity against pathogenic and spoilage micro-organisms, including fungi, and both Grampositive and Gram-negative bacteria. This study has also found that the Minimum Inhibition Concentration (MIC) of chitosan solution on Staphylococcus aureus was $3.97 \mathrm{mg} / \mathrm{ml}$ and the Minimum Inhibition Concentration of chitosan solution on Pseudomonas aeruginosa was $3.49 \mathrm{mg} / \mathrm{ml}$.
\end{abstract}

Keywords: Chitosan solution, Aspergillus niger, Staphylococcus aureus, Pseudomonas aeruginosa, MIC, food preservation.

\section{INTRODUCTION}

Microbial contamination, microbial growth and oxidation of lipids in foods during processing and storing are the major causes of food-borne illnesses and loss of shelf life. A considerable number of antimicrobial agents and antioxidants are therefore permitted by regulatory agencies to minimize the deterioration of food quality. But, synthetic preservatives are suspected of being responsible for some severe toxic effects. Thus, natural preservatives such as chitosan against different groups of microorganisms, such as bacteria, yeast and fungi have received considerable attention in recent years [1].

Chitosan, a polysaccharide biopolymer that combines a unique set of versatile physiochemical and biological characteristics which allow for a wide range of applications, of which has received attention as potential food preservative of natural origin due to its antibacterial and antifungal activities [2].

The major physical characteristics that determine the functional properties of the polymer are the molecular weight and the crystallinity [3]. Crystallinity is maximal for both chitin (i.e. $0 \%$ deacetylated) and fully deacetylated (i.e. $100 \%$ ) chitosan. Minimal crystallinity is achieved at intermediate degrees of deacetylation. Currently, chitin and chitosan are manufactured commercially in large scale from the outer shell of shrimps, lobsters and crabs.

Chitosan is insoluble at neutral and alkaline $\mathrm{pH}$, but forms water-soluble salts with inorganic and organic acids including glutamic, hydrochloric, lactic and acetic acids [4]. Upon dissolution in acidic media, the amino groups of the

*Address correspondence to this author at the School of Biotechnology, International University, Ho Chi Minh, Vietnam, Vietnam National University, Ho Chi Minh City, Vietnam; Fax: +84-83-7244271;

E-mail: nvtoan@hcmiu.edu.vn polymer become protonated rendering the molecule positively charged. The degree of acetylation represents the proportion of N-acetyl-D-glucosamine units with respect to the total number of units.

Chitosan exhibits a variety of physiochemical and biological properties; therefore it has been found numerous applications in various fields such as waste and water treatment, agriculture, fabric and textiles, cosmetics, nutritional enhancement, and food processing. In addition to its lack of toxicity and allergenicity, its biocompatibility, biodegradability and bioactivity make it a very attractive substance for diverse applications as a biomaterial in the biomedical, food, and chemical industries [5-7].

In this paper, successful application of chitosan in terms of microbial inhibition on some common food contaminating microbes is reported

\section{MATERIALS AND METHODS}

\section{Chitosan Sample}

Chitosan flake with DD of $90 \%$ was obtained by adapting Nguyen Van Toan's Method (2006), of which, the shrimp shells were preconditioned using dilute acetic acid [8].

\section{Chemical Preparation}

Acetic Acid 1\% (v/v) was prepared by taking $10.05 \mathrm{ml}$ of Glacial Acetic Acid (99.5\%) to the 1 liter-volumetric flask then distilled water was added until the volume reaches the bar of the volumetric flask. The flask was inverted several times to have the solution well mixed.

All the culture mediums were prepared by following the formulation of in Ronald M. Atlas [9]. with modification to match with the chemical availability at the laboratory.

In order to make agar medium, $1.6 \%(\mathrm{w} / \mathrm{v})$ agar was added to the broth and heated to boiling. All Petri dishes, test tubes, related equipments and culture medium must be 
sterilized by autoclaving for $15 \mathrm{~min}$ at $15 \mathrm{psi}$ pressure and $121^{\circ} \mathrm{C}$.

\section{Chitosan Film Preparation}

Chitosan film was prepared by dissolving $1 \%(\mathrm{w} / \mathrm{v})$ in $1 \mathrm{M}$ acetic acid. After filtration, the chitosan solution was cast onto a plastic plate, dried at $40^{\circ} \mathrm{C}$ and neutralized by soaking in $0.1 \mathrm{M} \mathrm{NaOH}$ for $30 \mathrm{~min}$ at ambient temperature. The membrane was then, subsequently washed thoroughly with distilled water and dried at $40^{\circ} \mathrm{C}$ for further examination of its quality. Chitosan membrane was tested for its thickness using a Mitutoyo micrometer, of which was adapted by Trung TS, et al, 2005.

\section{Chitosan Solutions Preparation}

Chitosan solution, after filtration and neutralization to $\mathrm{pH}$ 5.5, was titrated to determine the redundant acetic acid in the final solution. Solution of $\mathrm{NaOH} 0.1 \mathrm{M}$ was used as the titrant and phenolphthalein was used as color indicator. Sample of chitosan solution of $\mathrm{pH}$ at 5.5 was diluted ten times. $20 \mathrm{ml}$ of sample solution was taken to one flask for titration and 1-2 drops of phenolphthalein was added. The titration process was performed 5 times.

\section{Microbial Preparation}

Three kinds of microbes were purchased from the Institute of Drug Analysis, Ho chi Minh City, Vietnam. Staphylococcus aureus and Pseudomonas aeruginosa were cultured with LB broth in 100ml-Erlen flask for stocking and sub cultured every week on both LB agar and broth. Aspergillus niger was cultured on Sabouraud agar and the spore suspension was made by pouring $20 \mathrm{ml}$ of sterile physiological salt solution $(\mathrm{NaCl} 0.85 \%)$ into the 5-dayculture agar surface and gently scraped to remove the fungal spore [10]. The spore suspension was transferred into other sterile tube and keep in cool temperature for further use.

\section{MIC Estimations}

Sterilized Luria-Bertani broth was used for MIC estimation of Staphylococcus aureus and Pseudomonas aeruginosa [11]. The chitosan solution ( $\mathrm{pH}$ 5.6) was added into each test tube with the gradually increasing ratio: 1.1 up to 1.30. Sterilized water was added for balancing the volume in every test tube. The concentration of chitosan was calculated based on the whole volume of liquid inside each test tube.

Spore of Aspergillus niger was inoculated with chitosan solution $\mathrm{pH} 5.6$ at $37 \mathrm{oC}$ for testing the antifungal activity. The after-inoculated suspension was spread on Sabouraud agar again to observe the viability of the fungal spore.

$50 \mu 1$ of bacterial broth culture [12] was added to each test tube, and the chitosan solution $\mathrm{pH} 5.6$ was added with gradually increased of volume.

Contamination control test and test on effect of chitosan dissolving solvent were also conducted parallel with all the testing protocols. After loading all of the samples, all the test tubes were made vortex in 1 minute at $1000 \mathrm{rpm}$ to ensure the well mixing.

\section{Fruit Models}

Apples and bananas, purchased from the local market, were the subjects of the fruit testing models. Apples and bananas, after the transportation, were first washed with tap water, then with distilled water, and were sprayed with $70^{\circ}$ alcohol and let dried naturally at ambient temperature.

Two experiments were conducted. The first experiment included two groups of testing. In one group, chitosan solution at dilute concentration in aerosol form was sprayed into the skin of fruits. The other group was the control (with no treatment) for checking the storage time.

The second experiment also included two groups of testing. One group was infected by the spore of Aspergillus niger suspension in aerosol form, while the other was blank. Both groups were covered by the chitosan film for checking the spoilage.

\section{RESULTS AND DISCUSSION}

\section{Chitosan Films}

As shown in Fig. (1) and Table 1, the chitosan films were transparent and colorless. The average thickness of the film was $10 \pm 1 \mu \mathrm{m}$.
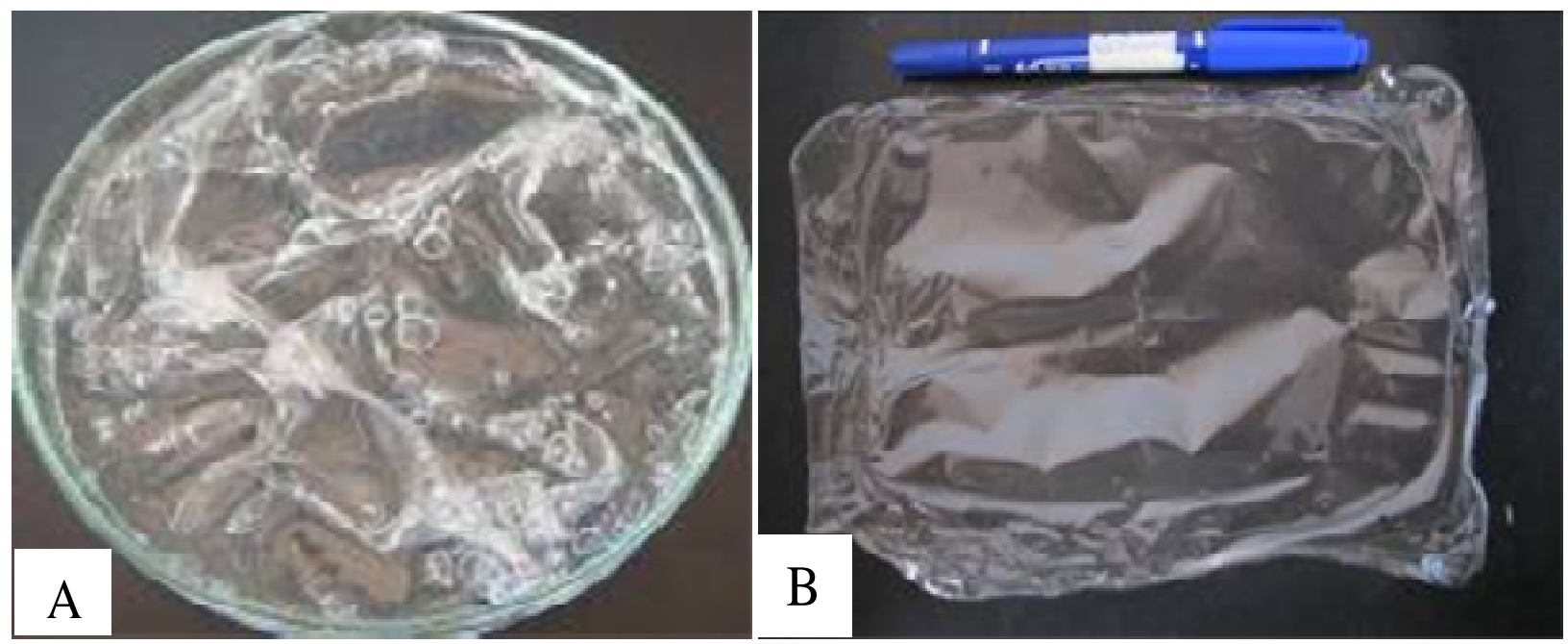

Fig. (1). Appearance of chitosan films before and after detaching (A) and drying in the lab (B) at ambient temperature. 
Table 1. Characteristics of Chitosan Membrane from the 90\% Deacetylated Chitosan

\begin{tabular}{|c|c|}
\hline Chitosan Film & Characteristic Index \\
\hline \hline Crystallinity $(\%)$ & $0.75 \pm 0.2$ \\
\hline Tensile strength $\left(\mathrm{N} / \mathrm{m}^{2}\right)$ & $2.35 \pm 0.4$ \\
\hline Moisture sorption $(\%)$ & $70 \pm 7.0$ \\
\hline Permeability constant $\left(\mathrm{x} 10^{-2} \mathrm{~cm}^{2} / \mathrm{h}\right)$ & $45.00 \pm 3$ \\
\hline
\end{tabular}

\section{MIC Estimations}

As shown in the Fig. (2), at the ratio of 1 to 2 (volume spore suspension / volume of chitosan solution), mixing on Sabouraud agar, after 15 days of incubation at $37^{\circ} \mathrm{C}$, chitosan solution express the ability to prevent spore germination and temporarily inhibit the growth of A. niger. When spore suspension was incubated with chitosan solution at $37^{\circ} \mathrm{C}$, without nutrition support (Sabouraud agar), after $12 \mathrm{hrs}$ of incubation, almost spores were terminated.

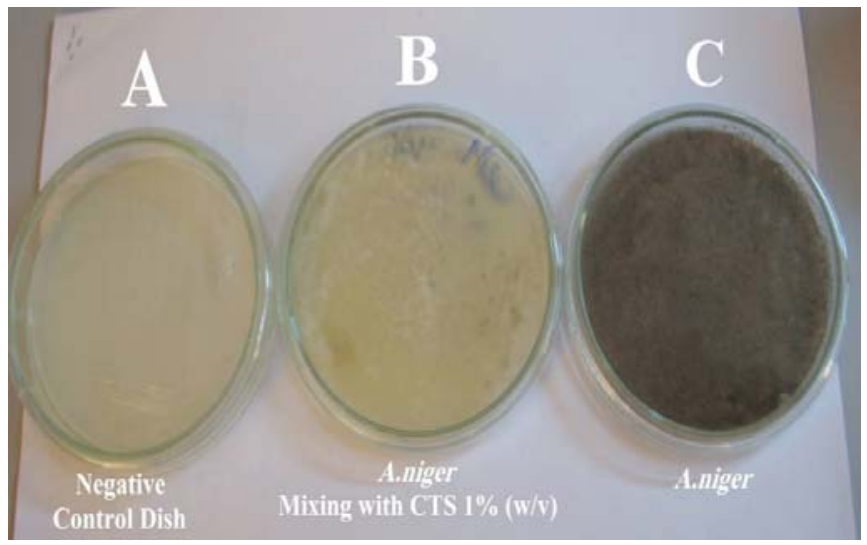

Fig. (2). The chitosan solution at $\mathrm{pH} 5.6$ can prevent the growth and spore germination of $A$. niger.

In the form of broth, the MIC estimation was done for Staphylococcus aureus by testing in a series of test tubes as shown in the Fig. (3).

Test tube 1 is the negative control with LB broth only. Test tube 2 is the LB broth with add the chitosan solution. Test tube 3 is the positive control with $S$. aureus innoculum added. Test tube 4 is the tube contain chitosan solution added with ratio 1 volume $S$. aureus innoculum versus 27 volume of chitosan solution. This tube showed the clear and transparency liquid, indicate that no bacterial growth inside. Test tube 5 is the tube with acetic acid $1 \%$. Test tube 6 contains $S$. aureus innoculum and the volume of redundant acetic acid in the test tube 4. From the above result, MIC of chitosan solution for Staphylococcus aureus is $3.97 \mathrm{mg} / \mathrm{ml}$.

In the Fig. (4), chitosan solution with the ratio of 1 volume innoculum versus 22 volume of chitosan solution can inhibit the growth of $P$. aeruginosa. Therefore, MIC of chitosan solution for Pseudomonas aeruginosa is $3.49 \mathrm{mg} / \mathrm{ml}$

Reportedly, chitosan causes extensive cell surface of microbes altered and covers the outer membrane with vesicular structures. Thus it can disrupt the properties of the outer membrane of the gram negative bacteria such as $P$. aeruginosa and $S$. aureus.

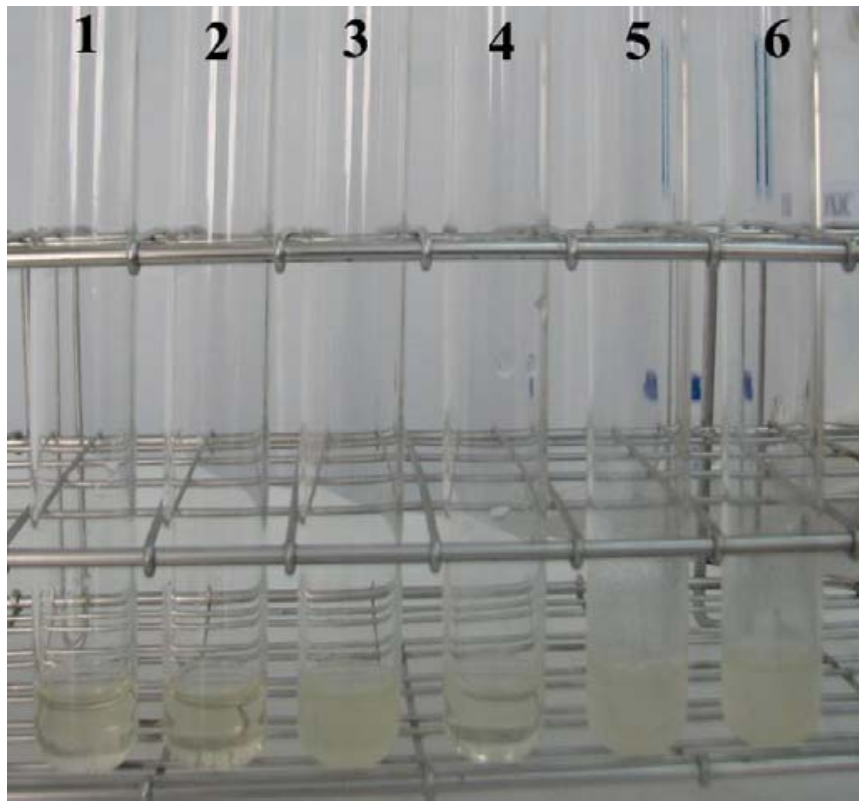

Fig. (3). Testing in broth culture of $S$. aureus.

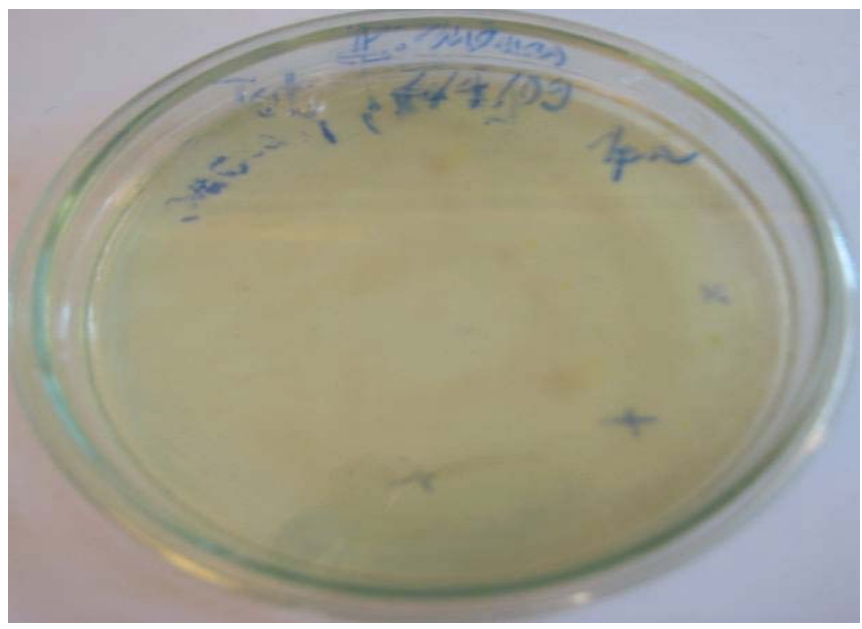

Fig. (4). Chitosan solution ( $\mathrm{pH}$ 5.6) added to the culture of $P$. aeruginosa on Lubria-Bertani agar express the strong antibacterial activity. The growth of Pseudomonas aeruginosa was completely inhibited.

\section{Fruit Models}

Figs. $(5,6)$ are showing a big difference in the fruit samples with and without the applications of chitosan solution. It can easily be seen that the bananas/ apples treated with chitosan solutions (Figs. 5a, 6a) still look fresh and in a good shape after 4 days of storage at ambient temperature while the bananas/ apples untreated with chitosan solutions (Figs. 5b, 6b) were infected with Pseudomonas aeruginosa and Staphylococcus aureus after 4 days of storage in the same condition. This also indicates that the prepared chitosan solutions have shown its strong inhibition on the presence, growth and development of some commonly mentioned food/fruits contaminating microbes. 


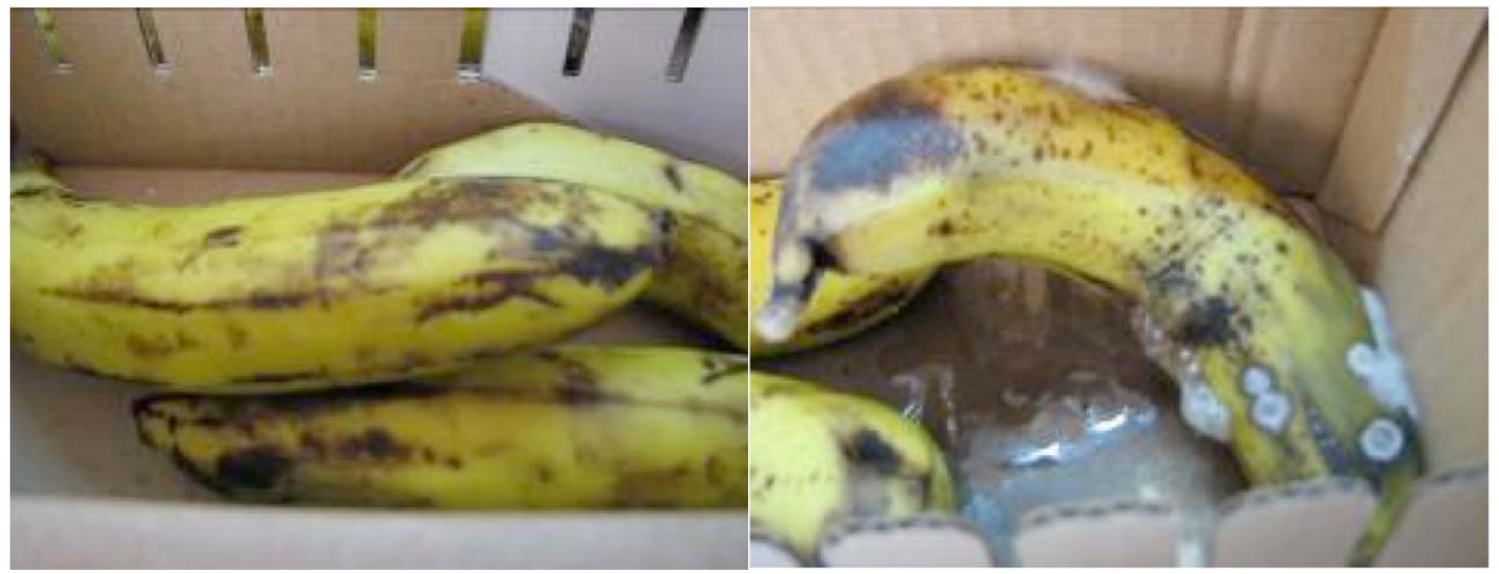

(a)

(b)

Fig. (5). Comparison between bananas treated with chitosan solution (a) and bananas without treatment (b) after 4-day-storage at ambient temperature.

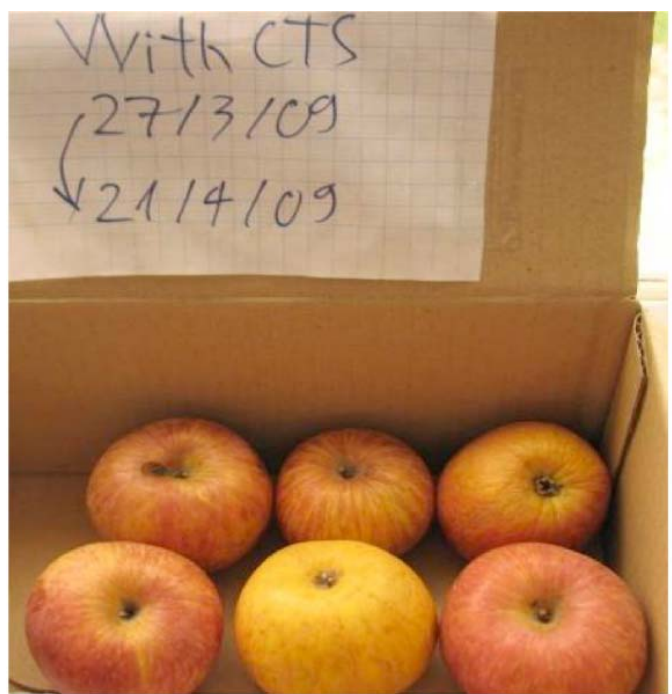

(a)

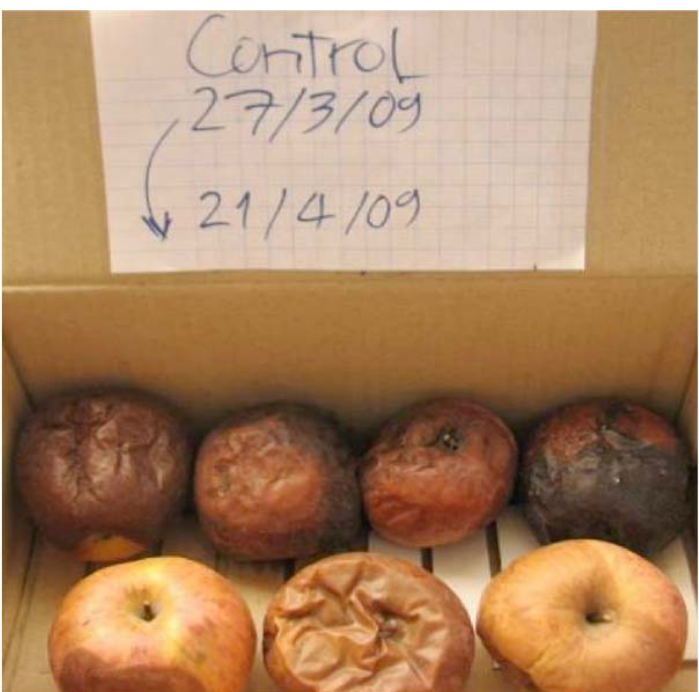

(b)

Fig. (6). Comparison between apples treated with chitosan solution and apples without treatment (control) after 1-month-storage at ambient tempearture.

\section{CONCLUSIONS}

From the above results, we can conclude that chitosan solution can inhibit the growth and development of A. niger, $S$. aureus, and $P$. aeruginosa. The testing model on fruit showed some defects on the chitosan film of which, caused by the air oxygen and light sensitivity characteristic of chitosan films. Minimum Inhibition Concentration (MIC) of chitosan solution on $S$. aureus was $3.97 \mathrm{mg} / \mathrm{ml}$. Minimum Inhibition Concentration of chitosan solution on $P$. aeruginosa was $3.49 \mathrm{mg} / \mathrm{ml}$.

\section{CONFLICT OF INTEREST}

The authors confirm that this article content has no conflict of interest.

\section{ACKNOWLEDGEMENTS}

Declared none.

\section{REFERENCES}

[1] Roller S. Chitosan disrupts the barrier properties of the outer membrane of Gram-negative bacteria. Int J Food Microbiol 2001; 71: 235-44.

[2] Sanford PA. Chitosan: commercial uses and potential applications In: G. Skjak-Braek, T. Anthonsen P, Sanford S, Eds. Chitin and Chitosan-Sources, Chemistry, Biochemistry, Physical Properties and Applications. London: Elsevier 1989; pp. 51-70.

[3] Trung TS, Thein-Han WW, Qui NT, Ng CH, Stevens WF. Functional characteristics of shrimp chitosan and its membranes as affected by the degree of deacetylation. Bioresour Technol 2006; 97(4): 659-63.

[4] Liu N, Chen XG, Park HJ, et al. Effect of MW and concentration of chitosan on antibacterial activity of Escherichia coli. Carbohydr Polym 2006; 64: 60-5.

[5] Senel S, McClure SJ. Potential applications of chitosan in veterinary medicine. Adv Drug Deliv Rev 2004; 56:1467-80.

[6] Rabea EI, Badawy MET, Stevens CV, Smagghe G, Steurbaut W. Chitosan as antimicrobial agent: applications and mode of action. Biomacromolecules 2003; 4:1457-65. 
[7] No HK, Meyers SP, Prinyawiwatkul W, Xu Z. Applications of chitosan for improvement of quality and shelf life of foods: a review. J Food Sci 2007; 72(5):R87-100.

[8] Toan NV, $\mathrm{Ng} \mathrm{CH}$, Aye KN, et al. Production of high-quality chitin and chitosan from preconditioned shrimp shells. J Chem Technol Biotechnol 2006; 81(7): 1113-8.

[9] Ronald MA. Handbook of Microbiological Media. UK: Taylor and Francis Group, LLC, CRC Press 2010
[10] Issam S, Adele MG, Adele CP, Stephane G, Veronique C. Chitosan Polymer as Bioactive Coating and film against Aspergillus niger Contamination. J Food Sci 2005; 70 (2): M100-4.

[11] Sherris JC. Antimicrobics and Chemotherapy of Bacterial and Viral Infections. In: Medical Microbiology. USA: Prentice-Hall International Inc 1990; pp. 197-9.

[12] Takahashi T. Growth inhibitory effect on bacteria of chitosan membranes regulated with deacetylation degree. Biochem Eng J 20078; 40: 485-91.

Received: May 9, 2013

Revised: June 27, 2013

Accepted: June 27, 2013

(C) Van Toan et al.; Licensee Bentham Open.

This is an open access article licensed under the terms of the Creative Commons Attribution Non-Commercial License (http://creativecommons.org/licenses/by$\mathrm{nc} / 3.0 /$ ) which permits unrestricted, non-commercial use, distribution and reproduction in any medium, provided the work is properly cited. 